\title{
The ergodic problem for some subelliptic operators with unbounded coefficients
}

\author{
Paola Mannucci@, Claudio Marchi and Nicoletta Tchou
}

\begin{abstract}
We study existence and uniqueness of the invariant measure for stochastic processes with degenerate diffusion, whose infinitesimal generators are linear subelliptic operators in the whole space $\mathbb{R}^{N}$ with possibly unbounded coefficients. Such a measure together with a Liouville-type theorem will play a crucial role in two applications: the ergodic problem studied through stationary problems with vanishing discount and the long time behavior of the solution to a parabolic Cauchy problem. In both cases, the constants will be characterized in terms of the invariant measure.
\end{abstract}

Mathematics Subject Classification. 35J70, 35H10, 35Q93, 49L25, 58F11.

Keywords. Subelliptic equations, Heisenberg group, Invariant measure, Viscosity solutions, Degenerate elliptic equations, Ergodic problem, Long time behavior.

\section{Introduction}

This paper is devoted to study with pde's methods both existence and uniqueness of the invariant measure of stochastic processes with degenerate diffusion, whose infinitesimal generators are linear subelliptic operators in the whole space $\mathbb{R}^{N}$ with possibly unbounded coefficients. The invariant measures play a crucial role in ergodicity, homogenization and large time behaviour of the value function associated to the process. These methods, based on optimal control theory and pde's arguments, were introduced in the 80's by Bensoussan and developed until nowadays (see the monograph [11] by Bensoussan and references therein).

We shall first tackle the case of the Heisenberg group as model problem; after we shall extend our techniques to other subelliptic operators. In the Heisenberg case, we consider the stochastic dynamics

$$
d X_{t}=b\left(X_{t}\right) d t+\sqrt{2} \sigma\left(X_{t}\right) d W_{t} \quad \text { for } t \in(0,+\infty), \quad X_{0}=x^{0} \in \mathbb{R}^{3}
$$


where, if $x=\left(x_{1}, x_{2}, x_{3}\right) \in \mathbb{R}^{3}$, the matrix $\sigma(x)$ has the form

$$
\sigma(x)=\left[\begin{array}{cc}
1 & 0 \\
0 & 1 \\
2 x_{2} & -2 x_{1}
\end{array}\right]
$$

(in other words, the columns of $\sigma$ are vectors generating the Heisenberg group) while $W_{t}$ is a two-dimensional Brownian motion.

Our principal aim is to prove, under suitable assumptions on the drift $b$ (see [2.7] below), existence and uniqueness of the invariant measure $m$ associated to the process (1.1).

Let us recall from [11] that a probability measure $m$ on $\mathbb{R}^{3}$ is an invariant measure for process (1.1) if, for each $u_{0} \in \mathbb{L}^{\infty}\left(\mathbb{R}^{3}\right)$, it satisfies

$$
\int_{\mathbb{R}^{3}} u(x, t) m(x) d x=\int_{\mathbb{R}^{3}} u_{0}(x) m(x) d x
$$

where $u(x, t)=\mathbb{E}_{x}\left(u_{0}\left(X_{t}\right)\right)$ is the solution to the parabolic Cauchy problem

$$
\left\{\begin{array}{l}
\partial_{t} u+\mathcal{L} u=0 \quad \text { in }(0,+\infty) \times \mathbb{R}^{3} \\
u(0, x)=u_{0}(x) \text { on } \mathbb{R}^{3}
\end{array}\right.
$$

where

$$
-\mathcal{L} u:=\operatorname{tr}\left(\sigma(x) \sigma^{T}(x) D^{2} u(x)\right)+b(x) \cdot D u(x)
$$

is the infinitesimal generator of process (1.1).

It is well known (see [11, Sect. II.4 and II.5] or [22, Section 5]) that the density of the probability $m$ (which, with a slight abuse of notation, we still denote by $m$ ) solves

$$
\mathcal{L}^{*} m=0, \quad \int_{\mathbb{R}^{3}} m d x=1 \quad \text { and } \quad m \geq 0,
$$

where $\mathcal{L}^{*} m$ is the adjoint operator to $\mathcal{L}$

$$
\begin{aligned}
\mathcal{L}^{*} m & =-\sum_{i, j} \partial_{i j}\left(\left(\sigma \sigma^{T}\right)_{i j} m\right)+\sum_{i} \partial_{i}\left(b_{i} m\right) \\
& =-\operatorname{tr}\left(\sigma \sigma^{T} D^{2} m\right)+\sum_{i} \partial_{i}\left(b_{i} m\right) .
\end{aligned}
$$

In the framework of locally strongly elliptic operators, Has'minskii [20, Sect. IV.4] (see also [29, Sect. 8.2]) established the existence of an invariant measure provided that there exists a bounded open set $U$ with smooth boundary such that

$$
\left\{\begin{array}{l}
\text { for any } x^{0} \in \mathbb{R}^{N} \backslash U, \text { the mean time } \tau \text { at which the path of } \\
\text { the diffusion given in (1.1) issuing from } x^{0} \text { reaches } U \text { is finite } \\
\text { and } \mathbb{E}_{x^{0}} \tau \text { is locally finite. }
\end{array}\right.
$$

In our case this result does not apply because the matrix $A:=\sigma \sigma^{T}$ with $\sigma$ given by $(1.2)$ is

$$
A(x)=\left[\begin{array}{ccc}
1 & 0 & 2 x_{2} \\
0 & 1 & -2 x_{1} \\
2 x_{2} & -2 x_{1} & 4\left(x_{1}^{2}+x_{2}^{2}\right)
\end{array}\right]
$$


and it is only positive semidefinite.

It is worth noticing (see $[7,17,28]$ ) that a sufficient condition for property $(1.5)$ is the existence of a Lyapunov-like function $w$ which satisfies, for some positive constants $k$ and $R_{0}$

$$
\mathcal{L} w \geq k \quad \text { for }|x| \geq R_{0} \quad \text { and } \quad w(x) \rightarrow+\infty \quad \text { as }|x| \rightarrow+\infty .
$$

As one can easily check the presence of the first order term is somehow 'crucial' for the existence of such a function. We will prove the existence of such Lyapunov function under suitable assumptions on the drift $b$ that include also the Ornstein-Uhlenbeck case (see [29]) where the operator is of the following type

$$
-\mathcal{L} u:=\operatorname{tr}\left(\sigma(x) \sigma^{T}(x) D^{2} u(x)\right)-\sum_{i=1}^{3} \gamma_{i} x_{i} \partial_{i} u(x)
$$

For the Ornstein-Uhlenbeck operator, we obtain the existence of a Lyapunov function when $\gamma_{i}>0$; this condition seems to be sharp (see example 2 below).

For ergodicity results based on probabilistic methods we refer to $[23,26]$ and references therein. We want to quote here the book of Arapostathis, Borkar, Ghosh [3] and the paper [9] where ergodicity is studied also for degenerate diffusions. The existence of a Lyapunov function is reminiscent of similar conditions (for instance, see: [29, Sect. 8.2] and the references therein), called "recurrence condition" in the probabilistic jargon.

Ichihara and Kunita [22] (see also [25]) proved the existence of an invariant measure for hypoelliptic processes as (1.1) which are constrained in a compact set. It is worth to recall that, in unbounded set the existence of an invariant measure may fail as it can be easily seen for (1.1) with $b=0$ and $\sigma=I$.

Dragoni, Kontis and Zegarlinski in [18] studied the ergodicity associated to Hörmander type generators strictly related to Lie group structure, even in the infinite dimensional case.

In this paper we want to establish existence and uniqueness of an invariant measure for process (1.1), namely for a process with the following features: it lies in an unbounded set and its infinitesimal generator is simultaneously degenerate and with unbounded coefficients. To this end we shall use only pure analytical arguments.

It is important to stress that, in the Heisenberg case, the principal part of $\mathcal{L} u$ can be written as $\sum_{i=1}^{2} X_{i}^{2} u$ where $X_{1}, X_{2}$, are the vector fields given by the columns of $\sigma$ and that they satisfy Hörmander condition: $X_{1}, X_{2}$, and their commutators of any order span $\mathbb{R}^{3}$ at each point $\left(x_{1}, x_{2}, x_{3}\right) \in \mathbb{R}^{3}$. In this case we have that $\left[X_{1}, X_{2}\right]=-4 \partial_{x_{3}}$. This property will play a crucial role in this paper since, as for the uniformly elliptic case, we have regularity, hypoellipticity, comparison and maximum principle [15].

The methods used in this work are strongly inspired by the lectures "Equations paraboliques et ergodicité" of P. L. Lions at Collège de France (2014-2015) [27] and by a unpublished manuscript by P. L. Lions and M. Musiela [28] (see also the paper of Cirant [17] for similar arguments). 
Actually, we shall consider the process

$$
d X_{t}^{\rho}=b\left(X_{t}^{\rho}\right) d t+\sqrt{2} \sigma_{\rho}\left(X_{t}^{\rho}\right) d W_{t},
$$

where $\sigma_{\rho}$ is the approximating matrix of $\sigma$ in (1.2)

$$
\sigma_{\rho}(x)=\left[\begin{array}{ccc}
1 & 0 & 0 \\
0 & 1 & 0 \\
2 x_{2} & -2 x_{1} & \rho
\end{array}\right]
$$

such that $A_{\rho}=\sigma_{\rho} \sigma_{\rho}^{T}$ is locally strictly positive, constrained in a bounded set $O_{n}$ suitably chosen.

Let us stress that, in our argument, it is not enough to approximate the matrix $A$ with any non-degenerate matrix $A_{\rho}$ but we also need that $A_{\rho}$ can be written as $\sigma_{\rho} \sigma_{\rho}^{T}$, where $\sigma_{\rho}$ is the diffusion matrix associated with a new Brownian motion.

Let us recall from [11] that the invariant measure $m_{\rho}^{n}$ of this process solves

$$
\mathcal{L}_{\rho}^{*} m_{\rho}^{n}=0 \quad \text { in } O_{n}
$$

coupled with a boundary condition of Neumann type, where

$$
-\mathcal{L}_{\rho}(u)=\operatorname{tr}\left(\sigma_{\rho}(x) \sigma_{\rho}^{T}(x) D^{2} u(x)\right)+b(x) \cdot D u(x)
$$

is an uniformly elliptic operator in $O_{n}$. Letting $n \rightarrow+\infty$, we obtain an invariant measure $m_{\rho}$ for the process (1.8) in the whole space; letting $\rho \rightarrow 0^{+}$, we get the desired invariant measure for (1.1). The Lyapunov function will play a crucial role in these limits: it will be used in order to prove that all the $m_{\rho}$ 's and $m$ are really measures (in other words, that the $m_{\rho}^{n}$ and the $m_{\rho}$ do not "disperse at infinity").

Moreover in this paper we also establish a Liouville type result. Similar result for semilinear operator without the drift term can be founded in the papers $[12,13,16]$ and references therein; in all these papers the nonlinear zeroth order term is the key ingredient whereas, in our setting, the crucial contribution is due to the drift. After finishing this paper we became aware of [6] where a Liouville property is studied for quasilinear hypoelliptic operators.

We shall use the invariant measure and the Liouville property in two classical applications: an ergodic problem and the long time behaviour of a Cauchy problem. For the former problem we consider the family of equations

$$
\delta u_{\delta}-\operatorname{tr}\left(\sigma(x) \sigma^{T}(x) D^{2} u_{\delta}\right)-b(x) D u_{\delta}=f(x) \text { in } \mathbb{R}^{3},
$$

where $\delta>0$ and we shall prove that, as $\delta \rightarrow 0, \delta u_{\delta}$ converges to a constant $\lambda$, called "ergodic" constant. Let us stress that the differential operator in the ergodic problem coincides with the infinitesimal generator $\mathcal{L}$ of process (1.1).

We recall that the study of ergodic problems for equations with periodic, uniformly elliptic, operators has been addressed in $[4,10]$ while, for periodic, possibly degenerate (still satisfying the Hörmander condition) operators, we refer the reader to the papers $[1,2]$.

The main difficulties in our problem are the lack of periodicity and the degeneracy of the operator. We shall overcome these issues using some techniques introduced by [7] for an elliptic operator on the whole space. Moreover, 
we shall give an explicit formula for the ergodic constant $\lambda$ in terms of the invariant measure for (1.1).

In the latter application we consider the following Cauchy problem:

$$
u_{t}+\mathcal{L} u=0 \quad \text { in }(0,+\infty) \times \mathbb{R}^{3}, \quad u(0, x)=f(x) \quad \text { on } \mathbb{R}^{3},
$$

where $\mathcal{L}$ is the operator defined in (1.4). We will prove that, as $t \rightarrow+\infty$, the solution $u$ converges to a constant $\Lambda$ which will be characterized in terms of the invariant measure.

Finally, we shall show how to extend our previous results to other degenerate operators satisfying Hörmander condition with possibly unbounded coefficients.

Our future purpose [30] is to use the ergodic problem to study the homogenization problem

$$
-\epsilon \operatorname{tr}\left(\sigma\left(\frac{x}{\epsilon}\right) \sigma^{T}\left(\frac{x}{\epsilon}\right) D^{2} u_{\epsilon}\right)-b\left(\frac{x}{\epsilon}\right) \cdot D u_{\epsilon}+f\left(x, \frac{x}{\epsilon}\right)+a u_{\epsilon}=0 \text { in } \mathbb{R}^{3},
$$

where $\sigma$ has the form (1.2). In this case the approximated cell problem formally coincides with the problem (1.9).

For the study of homogenization problems for periodic, possibly nonlinear, degenerate (still satisfying the Hörmander condition) operators, we refer the reader to the papers $[1,14,31]$.

This paper is organized as follows: Sect. 2 contains the main result of the paper: we find conditions on the drift $b$ such that a Lyapunov function does exist and by means of this function we prove existence and uniqueness of an invariant measure associated to our process. In Sect. 3, we establish a Liouville type result assuming the existence of a Lyapunov-like function. Section 4 is devoted to our applications: in Sect. 4.1 we study the ergodic problem through stationary problems with vanishing discount, while in Sect. 4.2 we consider the long time behaviour of a Cauchy problem. In Sect. 5 we generalize the previous results to a more general class of subelliptic operators, encompassing e.g. the Grushin one. The Appendix contains a condition equivalent to (1.7) which will be useful to manage the Lyapunov function founded in Sect. 2.

\section{Existence and uniqueness of the invariant measure}

This section is devoted to the invariant measure for process (1.1). Let us recall (see [28] or Proposition 2.1 below) that, when the matrix associated to the infinitesimal generator $\mathcal{L}$ is a strictly definite positive matrix, a sufficient condition for the existence of an invariant measure is given by: there exists a Lyapunov-like function such that

$$
\begin{aligned}
& w \in C^{\infty}\left(B_{0}^{C}\right) \cap C^{0}\left(\mathbb{R}^{3}\right) \\
& \mathcal{L} w \geq 1, \quad \text { in } B_{0}^{C} \\
& w \geq 0 \quad \text { in } B_{0}^{C}, \quad w=0 \quad \text { on } \partial B_{0},
\end{aligned}
$$

where $B_{0}$ is a ball centered in 0 with suitable radius (for less regular functions $w$, we refer to $[28])$. 
In our case, the matrix $A=\sigma \sigma^{T}$ in (1.6) is degenerate at any point and its rank is 2 . In order to overcome this issue, for $\rho>0$, we introduce the approximating operators

$$
\mathcal{L}_{\rho} w:=-\operatorname{tr}\left(A_{\rho}(x) D^{2} w\right)-b(x) D w,
$$

where

$$
A_{\rho}(x)=\left[\begin{array}{ccc}
1 & 0 & 2 x_{2} \\
0 & 1 & -2 x_{1} \\
2 x_{2} & -2 x_{1} & 4\left(x_{1}^{2}+x_{2}^{2}\right)+\rho^{2}
\end{array}\right]=\sigma(x) \sigma^{T}(x)+\left[\begin{array}{ccc}
0 & 0 & 0 \\
0 & 0 & 0 \\
0 & 0 & \rho^{2}
\end{array}\right] .
$$

In the following lemma we collect some useful properties of $\mathcal{L}_{\rho}$.

Lemma 2.1. The matrix $A_{\rho}(x)$ is locally strictly positive definite (namely, for any compact $K \subset \mathbb{R}^{3}$, there holds $\lambda A_{\rho}(x) \lambda^{T} \geq \nu(x)|\lambda|^{2}$ for any $x \in K$, with $\nu(x) \geq a(K, \rho)>0$ ) and it is positive definite in $\mathbb{R}^{3}$.

Moreover, the matrix

$$
\sigma_{\rho}(x)=\left[\begin{array}{ccc}
1 & 0 & 0 \\
0 & 1 & 0 \\
2 x_{2} & -2 x_{1} & \rho
\end{array}\right]
$$

fulfills

$$
A_{\rho}(x)=\sigma_{\rho}(x) \sigma_{\rho}^{T}(x) .
$$

Proof. Set $\alpha=4\left(x_{1}^{2}+x_{2}^{2}\right)+\rho^{2}+1$. The eigenvalues of $A_{\rho}$ are

$$
\lambda_{1}=1, \quad \lambda_{2,3}=\frac{\alpha \pm \sqrt{\alpha^{2}-4 \rho^{2}}}{2} .
$$

It is easy to remark that $\lambda_{2} \geq \frac{1}{2}$.

The last eigenvalue is $\lambda_{3}=\frac{2 \rho^{2}}{\alpha+\sqrt{\alpha^{2}-4 \rho^{2}}}>\frac{\rho^{2}}{\alpha}$ hence, for any fixed $R>0$, if $x_{1}^{2}+x_{2}^{2} \leq R^{2}, \alpha \leq 4 R^{2}+\rho^{2}+1$ and $\lambda_{3}>\frac{\rho^{2}}{4 R^{2}+\rho^{2}+1}>0$.

The last part of the statement follows by a simple computation, so we shall omit its proof.

Remark 2.1. From (2.5), beside being uniformly elliptic, the operator $-\mathcal{L}_{\rho}$ is also the infinitesimal generator of the stochastic process

$$
d X_{t}^{\rho}=b\left(X_{t}^{\rho}\right) d t+\sqrt{2} \sigma_{\rho}\left(X_{t}^{\rho}\right) d \tilde{W}_{t},
$$

where $\sigma_{\rho}$ is defined in (2.4) and $\tilde{W}_{t}$ is a three-dimension Brownian motion whereas our starting process (1.1) contains a two-dimension Brownian motion.

Now, we want to prove that, for some classes of drifts $b$, there exists a function $w$ satisfying $(2.1)$ with $\mathcal{L}$ replaced by $\mathcal{L}_{\rho}$. To this end, we consider a continuous drift $b=\left(b_{1}, b_{2}, b_{3}\right)$ such that

$$
b_{i}(x)=b_{i}\left(x_{i}\right), \quad \begin{cases}b_{i}\left(x_{i}\right) \leq-\frac{C_{i}}{\left|x_{i}\right|^{1-\alpha}} & \text { for } x_{i} \geq R \\ b_{i}\left(x_{i}\right) \geq \frac{C_{i}}{\left|x_{i}\right|^{1-\alpha}} & \text { for } x_{i} \leq-R\end{cases}
$$

for some constants $\alpha \geq 0, R>0$ and $C_{i}>0(i=1,2,3)$. 
Note that Lemma 2.2 below holds also for $\rho=0$ then we have a Lyapunovlike function $w$ (i.e. satisfying condition [2.1]) also for the degenerate starting problem where $\mathcal{L}$ is given by (3.1).

Conditions similar to (2.7) were obtained in [28] with $\sigma=I$ the identity matrix.

Lemma 2.2. Assume $\sigma$ as in (1.2) and $b$ as in (2.7). Then the function

$$
w(x):=\frac{\left(x_{1}^{4}+x_{2}^{4}\right)}{12}+\frac{x_{3}^{2}}{2}
$$

satisfies

$$
\mathcal{L}_{\rho} w \geq 1 \quad \text { in } \overline{B\left(0, R_{0}\right)}{ }^{C}, \quad w \geq 0 \quad \text { in } \overline{B\left(0, R_{0}\right)}{ }^{C}, \quad \lim _{|x| \rightarrow \infty} w=\infty
$$

for $R_{0}$ sufficiently large, $\rho$ sufficiently small and

(i) either $\alpha>0$

(ii) or $\alpha=0$ and sufficiently large $C_{i}$.

Proof. Using (2.8), we obtain

$$
\mathcal{L}_{\rho} w=-5\left(x_{1}^{2}+x_{2}^{2}\right)-\rho^{2}-\frac{1}{3}\left(b_{1} x_{1}^{3}+b_{2} x_{2}^{3}\right)-b_{3} x_{3} .
$$

We denote $K_{i}:=\max _{x_{i} \in[-R, R]}\left|b_{i}\left(x_{i}\right)\right|$.

Case (i). Assume $\alpha>0$. We want to prove that there exists $R_{0}$ such that $\mathcal{L}_{\rho} w>$ 1 in $\overline{B\left(0, R_{0}\right)}$ for $\rho$ sufficiently small. To this end, we split the arguments in several cases.

(I) If $\left|x_{i}\right| \geq R$ for any $i \in\{1,2,3\}$, then

$$
\mathcal{L}_{\rho} w \geq x_{1}^{2}\left(-5+C_{1}\left|x_{1}\right|^{\alpha} / 3\right)+x_{2}^{2}\left(-5+C_{2}\left|x_{2}\right|^{\alpha} / 3\right)+C_{3}\left|x_{3}\right|^{\alpha}-\rho^{2} .
$$

Hence, for $\left|x_{1}\right|,\left|x_{2}\right|>R_{1}:=\max \left\{\left(15 / C_{1}\right)^{1 / \alpha},\left(15 / C_{2}\right)^{1 / \alpha}, R\right\},\left|x_{3}\right| \geq$ $R_{3}:=\max \left\{C_{3}^{-1 / \alpha}, R\right\}$, we get: $\mathcal{L}_{\rho} w \geq 1$ for $\rho$ sufficiently small.

(II) If $\left|x_{1}\right|,\left|x_{2}\right| \leq R_{1}$ and $\left|x_{3}\right| \geq R$, then

$$
\mathcal{L}_{\rho} w \geq-10 R_{1}^{2}-\rho^{2}-R^{3}\left(K_{1}+K_{2}\right) / 3+C_{3}\left|x_{3}\right|^{\alpha}
$$

(here, we used the relation: $-b_{i} x_{i}^{3} \geq 0$ for $\left.\left|x_{i}\right| \in\left[R, R_{1}\right], i=1,2\right)$. Hence, for $\left|x_{3}\right| \geq \tilde{R}_{3}$ with $\tilde{R}_{3}$ sufficiently large, taking $\rho$ sufficiently small, we get $\mathcal{L}_{\rho} w \geq 1$.

(III) If $\left|x_{1}\right| \leq R_{1},\left|x_{2}\right| \geq R_{1}$ and $\left|x_{3}\right| \geq R$ (and similarly, for $\left|x_{1}\right| \geq R_{1}$, $\left|x_{2}\right| \leq R_{1}$ and $\left.\left|x_{3}\right| \geq R\right)$, then

$$
\mathcal{L}_{\rho} w \geq-5 R_{1}^{2}+x_{2}^{2}\left(-5+\left|x_{2}\right|^{\alpha} / 3\right)-\rho^{2}-K_{1} R^{3} / 3+C_{3}\left|x_{3}\right|^{\alpha} .
$$

Hence, for $\left|x_{3}\right| \geq \tilde{R}_{3}$, we get $\mathcal{L}_{\rho} w \geq 1$ for $\rho$ sufficiently small.

(IV) If $\left|x_{1}\right| \leq R_{1},\left|x_{2}\right|>R,\left|x_{3}\right|<\tilde{R}_{3}$ (and similarly for $\left|x_{1}\right|>R,\left|x_{2}\right| \leq R_{1}$, $\left.\left|x_{3}\right|<\tilde{R}_{3}\right)$, then

$$
\mathcal{L}_{\rho} w \geq\left|x_{2}\right|^{2}\left(-5+C_{2}\left|x_{2}\right|^{\alpha} / 3\right)-5 R_{1}^{2}-\rho^{2}-K_{1} R^{2} / 3-K_{3} R .
$$

Hence, for $\left|x_{2}\right|>R_{1}$, we get $\mathcal{L}_{\rho} w \geq 1$ for $\rho$ sufficiently small. 
In conclusion, gluing together all these cases, we accomplish the proof for $\alpha>0$.

Case (ii). Assume $\alpha=0$; we want to prove that there exist some constants $C_{i}$ and a radius $R_{0}$ such that $\mathcal{L}_{\rho} w \geq 1$ in $\overline{B\left(0, R_{0}\right)} C$.

(I) If $\left|x_{i}\right|>R$ for any $i=1,2,3$, then $\mathcal{L}_{\rho} w \geq x_{1}^{2}\left(-5+\frac{1}{3} C_{1}\right)+x_{2}^{2}(-5+$ $\left.\frac{1}{3} C_{1}\right)+C_{3}-\rho^{2}$; hence, for $C_{1}, C_{2}>15, C_{3}>1$, we have $L_{\rho} w>1$ for $\rho$ sufficiently small.

(II) If $\left|x_{i}\right|<R$ for $i=1,2$ and $\left|x_{3}\right|>R$, then $\mathcal{L}_{\rho} w \geq-10 R^{2}-R^{3}\left(K_{1}+\right.$ $\left.K_{2}\right) / 3-\rho^{2}+C_{3}$; hence, for $C_{3}>10 R^{2}+R^{3}\left(K_{1}+K_{2}\right) / 3+1$, we have $\mathcal{L}_{\rho} w>1$ for $\rho$ sufficiently small.

(III) If $\left|x_{1}\right|<R,\left|x_{2}\right|>R$ and $\left|x_{3}\right|>R$ (and similarly, for $\left|x_{1}\right| \geq R,\left|x_{2}\right| \leq R$ and $\left.\left|x_{3}\right| \geq R\right)$, then $\mathcal{L}_{\rho} w \geq-5 R^{2}+x_{2}^{2}\left(-5+C_{2} / 3\right)-R^{2} K_{1} / 3-\rho^{2}+C_{3}$, hence, for $C_{2}>15, C_{3}$ sufficiently large and $\rho$ sufficiently small, we have $\mathcal{L}_{\rho} w>1$.

(IV) If $\left|x_{1}\right| \leq R,\left|x_{2}\right|>R,\left|x_{3}\right|<R$ (and similarly for $\left|x_{1}\right|>R,\left|x_{2}\right| \leq R$, $\left.\left|x_{3}\right|<R\right)$, then $\mathcal{L}_{\rho} w \geq-5 R^{2}+x_{2}^{2}\left(-5+C_{2} / 3\right)-K_{1} R^{2}-\rho^{2}-K_{3} R$; hence, for $C_{2}>15,\left|x_{2}\right|$ sufficiently large and $\rho$ sufficiently small, we have $\mathcal{L}_{\rho} w>1$.

Remark 2.2. Stronger sufficient condition on $b_{i}$ for the existence of a Lyapunovlike function $w$ satisfying condition (2.1) could be found using $w(x):=\log \left(\left(x_{1}^{2}+\right.\right.$ $\left.\left.\left.x_{2}^{2}\right)^{2}+x_{3}^{2}\right)\right)$.

Example 2.1 The Ornstein-Uhlenbeck drift.

Let $b(x)=-\gamma x, \gamma=\left(\gamma_{1}, \gamma_{2}, \gamma_{3}\right)$, this is the model Ornstein-Uhlenbeck case (for further properties of this operator see [29]). In the model case (non degenerate operator with bounded coefficients) of the Laplace operator i.e. $\mathcal{L} u=-\Delta u+\gamma x \cdot D u$, there exists a natural Lyapunov function $r^{2}:=\sum_{i} x_{i}^{2}$ if all the $\gamma_{i}$ are positive (see also [6, Corollary 2.3] for Hamilton-Jacobi-Bellman operators).

In the complete degenerate case $\mathcal{L} u=\gamma x \cdot D u$, the function $r^{2}=\sum_{i} x_{i}^{2}$ is a Lyapunov function if all the $\gamma_{i}$ are positive. But $r^{2}$ is a Lyapunov function for the Heisenberg operator (1.4) only if $\gamma_{1}>4, \gamma_{2}>4, \gamma_{3}>0$. The choice of $w$ in (2.8) as a Lyapunov function for the operator (1.4) allows us to apply our results to a much more general Ornstein-Uhlenbeck drift, i.e. to the case where all the $\gamma_{i}$ are positive. Let us also remark that in other strongly elliptic case with unbounded coefficients (still with linear growth at infinity) we cannot find, in general, so weak assumptions on the $\gamma_{i}$.

In the next proposition we will establish the existence of an invariant measure $m_{\rho}$ of the approximating process (2.6). This measure will be used in the main theorem of this paper when the invariant measure for the process (1.1) will be obtained as the limit of $m_{\rho}$ as $\rho \rightarrow 0$.

Proposition 2.1. Let $\sigma_{\rho}(x)$ be defined by (2.4) and $b(x)$ be a Lipschitz function. Assume that

(i) either $b$ satisfies (2.7) with $\alpha>0$, 
(ii) or $b$ is smooth and satisfies (2.7) with $\alpha=0$ and $C_{i}$ sufficiently large.

Then, there exists a unique invariant probability measure $m_{\rho}$ on $\mathbb{R}^{3}$ for the process (2.6).

Proof. As proved in Lemma 2.1 the operator $\mathcal{L}_{\rho}$ is uniformly elliptic in each bounded set (but the ellipticity constant degenerates in the whole $\mathbb{R}^{3}$ ). We adapt some techniques introduced by [28] (see also [17] for similar arguments), by considering approximate problems in domains $O_{n}$ such that $O_{n} \nearrow \mathbb{R}^{3}$ as $n \rightarrow+\infty$.

We claim that, in both cases, there exists a function $\bar{w}$ such that

$$
\bar{w} \in C^{\infty}\left(\mathbb{R}^{3}\right), \quad \mathcal{L}_{\rho} \bar{w}+\chi \bar{w}=\phi \quad \text { in } \mathbb{R}^{3}, \quad \lim _{|x| \rightarrow+\infty} \bar{w}=\infty
$$

where $\chi \in C_{0}^{\infty}$ and $\phi \in C^{\infty}$ are suitable functions such that, $\chi>0$ on $B_{0}, \operatorname{supp} \chi=\bar{B}_{0}\left(B_{0}\right.$ is a suitable bounded open set $)$ and $\lim _{|x| \rightarrow \infty} \phi=\infty$. Actually, in case $(i)$, the function $w$ defined in (2.8) satisfies (2.10). Moreover, let us recall (see [27] or Lemma A.1 in the Appendix) that, for $b$ smooth, condition (2.9) is equivalent to condition (2.10); in particular, by Lemma 2.2, we deduce the existence of a function $\bar{w}$ as in (2.10) also in case (ii). Hence, our claim is completely proved.

Fix a function $\bar{w}$ as in (2.10) and define $O_{n}:=\left\{x \in \mathbb{R}^{3} \mid \bar{w}(x)<M_{n}\right\}$ where $M_{n} \rightarrow+\infty$ if $n \rightarrow+\infty$ and $M_{n}$ is not a critical value of $\bar{w}$. Since $\bar{w} \rightarrow+\infty$ if $x \rightarrow+\infty$ then $O_{n}$ are bounded and smooth and $O_{n} \nearrow \mathbb{R}^{3}$. Fix $\rho>0$ and $n$, the results by Bensoussan [11, Section 4] ensure that there exists an unique invariant measure $m_{\rho}^{n}$ associated to the diffusion process $X_{t}^{\rho}$ in $O_{n}$ with reflecting boundary whose infinitesimal generator is $L_{\rho}$ in $O_{n}$ with boundary conditions

$$
\sum_{i, j}\left(a_{\rho}\right)_{i j} \frac{\partial u}{\partial \nu_{j}}=0 \quad \text { on } \partial O_{n}
$$

where $\nu$ denotes the unit outward normal to $\partial O_{n}$ and the matrix $A_{\rho}=\left(a_{\rho}\right)_{i j}=$ $\sigma_{\rho} \sigma_{\rho}^{T}$ as in Lemma 2.1 .

The invariant measure $m_{\rho}^{n}$ satisfies the problem

$$
\begin{aligned}
& \mathcal{L}_{\rho}^{*} m_{\rho}^{n}:=-\sum_{i, j} \frac{\partial^{2}\left(\left(a_{\rho}\right)_{i j} m_{\rho}^{n}\right)}{\partial x_{i} \partial x_{j}}+\sum_{i} \frac{\partial\left(b_{i} m_{\rho}^{n}\right)}{\partial x_{i}}=0 \quad \text { in } O_{n} \\
& \sum_{i j} \nu_{i}\left(\frac{\partial\left(\left(a_{\rho}\right)_{i j} m_{\rho}^{n}\right)}{\partial x_{j}}-b_{i} m_{\rho}^{n}\right)=0 \quad \text { on } \partial O_{n} \\
& \int_{O_{n}} m_{\rho}^{n}=1, \quad m_{\rho}^{n}>0 .
\end{aligned}
$$


We have to prove that, as $n \rightarrow+\infty, m_{\rho}^{n}$ converges in some sense to $m_{\rho}$ invariant measure to the process with generator $\mathcal{L}_{\rho}$, i.e. $m_{\rho}$ solves

$$
\begin{aligned}
\mathcal{L}_{\rho}^{*} m_{\rho} & :=-\sum_{i, j} \frac{\partial^{2}\left(\left(a_{\rho}\right)_{i j} m_{\rho}\right)}{\partial x_{i} \partial x_{j}}+\sum_{i} \frac{\partial\left(b_{i} m_{\rho}\right)}{\partial x_{i}}=0 \quad \text { in } \mathbb{R}^{3} \\
\int_{\mathbb{R}^{3}} m_{\rho} & =1, \quad m_{\rho} \geq 0 .
\end{aligned}
$$

From Prohorov Theorem and the fact that $\int_{O_{n}} m_{\rho}^{n}=1$ we know that $m_{\rho}^{n} \rightarrow m_{\rho}$ as $n \rightarrow+\infty$ (possibly passing to a subsequence). We prove now that $\int_{\mathbb{R}^{3}} m_{\rho}=$ 1. Multiplying Eq. (2.11) by $\bar{w}$ defined in (2.10), integrating on $O_{n}$ and taking into account (2.12) we obtain

$$
0=\int_{O_{n}} \mathcal{L}_{\rho}^{*} m_{\rho}^{n} \bar{w}=\int_{O_{n}} m_{\rho}^{n} \mathcal{L}_{\rho} \bar{w}+\int_{\partial O_{n}} m_{\rho}^{n} \sum_{i, j}\left(a_{\rho}\right)_{i j} \frac{\partial \bar{w}}{\partial x_{i}} \nu_{j}
$$

Since $\bar{w}=M_{n}$ on $\partial O_{n}$ and $\bar{w}<M_{n}$ on $O_{n}$, we have $\frac{\partial \bar{w}}{\partial x_{i}}=\frac{\partial w}{\partial \nu} \nu_{i}$ and $\frac{\partial \bar{w}}{\partial \nu} \geq 0$ on $\partial O_{n}$. Then, there holds

$$
0=\int_{O_{n}} m_{\rho}^{n} \mathcal{L}_{\rho} \bar{w}+\frac{\partial \bar{w}}{\partial \nu} \int_{\partial O_{n}} m_{\rho}^{n} \sum_{i, j}\left(a_{\rho}\right)_{i j} \nu_{i} \nu_{j},
$$

and, since $\sum_{i, j}\left(a_{\rho}\right)_{i j} \nu_{i} \nu_{j} \geq 0$, we obtain $\int_{O_{n}} m_{\rho}^{n} \mathcal{L}_{\rho} \bar{w} \leq 0$. Hence, there holds

$$
\int_{O_{n}} m_{\rho}^{n} \mathcal{L}_{\rho} \bar{w}=\int_{O_{n}}(\phi-\chi \bar{w}) m_{\rho}^{n} \leq 0,
$$

and

$$
\int_{O_{n}} \phi m_{\rho}^{n} \leq \int_{\text {supp } \chi} \chi \bar{w} m_{\rho}^{n} \leq C
$$

where $C$ is a positive constant independent of $n$. Let us extend $m_{\rho}^{n}$ by zero outside $O_{n}$, and still call it $m_{\rho}^{n}$, then

$$
\int_{\mathbf{R}^{3}} \phi m_{\rho}^{n} \leq C
$$

where $C$ is a positive constant independent of $n$.

Since $\lim _{|x| \rightarrow+\infty} \phi(x)=+\infty$, for any $N$ there exists a $R_{N}$ such that $\phi(x)>N$ on $B_{R_{N}}^{C}$. Hence, from (2.14), we infer

$$
\int_{B_{R_{N}}^{C}} m_{\rho}^{n} \leq \frac{C}{N}
$$

Since $\int_{\mathbf{R}^{3}} m_{\rho}^{n}=1$, then from $(2.15)$ we deduce

$$
\int_{B_{R_{N}}} m_{\rho}^{n} \geq 1-\frac{C}{N}
$$

and from the weak convergence of $m_{\rho}^{n}$ to $m_{\rho}$, we have

$$
\int_{B_{R_{N}}} m_{\rho} \geq 1-\frac{C}{N} ;
$$


hence, letting $N \rightarrow+\infty$, we obtain that $\int_{\mathbb{R}^{3}} m_{\rho}=1$.

Moreover from the local regularity $W^{2, p}$ for any $p>1$ of $m_{\rho}^{n}$ since $m_{\rho}^{n}$ solves Eq. (2.11), passing to the limit we easily obtain that $m_{\rho}$ solves Eq. (2.13).

Remark 2.3. Under the hypotheses of Proposition 2.1, the condition of strict ellipticity in the compact subsets of $\mathbb{R}^{N}$ is sufficient to deduce from (2.9) the existence of the invariant measure $m_{\rho}$ (see [20, Theorem IV.4.1] under their assumption B.1). Nevertheless we gave the proof of Proposition 2.1 because it is purely analytic and for the sake of completeness.

Now we want to prove that, as $\rho \rightarrow 0^{+}, m_{\rho}$ converges in some sense to $m$, invariant measure to the process $(1.1)$, solving

$$
\mathcal{L}^{*} m=0, \quad \int_{\mathbb{R}^{3}} m=1 \quad \text { and } m \geq 0 .
$$

Theorem 2.1. Let $\sigma$ be defined by (1.2) and $b$ be a Lipschitz function. Assume that either $b$ satisfies (2.7) with $\alpha>0$ or $b$ is smooth and satisfies (2.7) with $\alpha=0$ and $C_{i}$ sufficiently large. Then, there exists a unique invariant probability measure $m$ on $\mathbb{R}^{3}$ for the process (1.1).

Proof. The existence of the invariant measure it is obtained proving that, as $\rho \rightarrow 0^{+}$, the invariant measure $m_{\rho}$ of Proposition 2.1 converges to the measure $m$ associated to the process (1.1). We proceed analogously to Proposition 2.1. The measure $m_{\rho}$ satisfies the following conditions:

$$
\mathcal{L}_{\rho}^{*} m_{\rho}=0 \quad \text { in } \mathbb{R}^{3}, \quad \int_{\mathbb{R}^{N}} m_{\rho}=1, \quad m_{\rho} \geq 0 .
$$

We know that $m_{\rho} \rightarrow m$ as $\rho \rightarrow 0$ (at least for a subsequence) where $m$ is a measure. We have to prove that $m$ is an invariant measure to the process (1.1) i.e. that $m$ solves $(2.16)$.

From condition (2.9), arguing as in the proof of Proposition 2.1 to get (2.10), we know that there exist smooth functions $\chi$ and $\phi$ such that $\bar{w}$ satisfies $\mathcal{L}_{\rho} \bar{w}+\chi \bar{w}=\phi$, in $\mathbb{R}^{3}, \bar{w}$ and $\phi$ diverge to $+\infty$ if $|x| \rightarrow+\infty$ and $\chi$ has compact support.

Multiplying Eq. (2.13) by such $\bar{w}$ and integrating on $\mathbb{R}^{3}$ we obtain

$$
0=\int_{\mathbb{R}^{3}} \mathcal{L}_{\rho}^{*} m_{\rho} \bar{w}=\int_{\mathbb{R}^{3}} \mathcal{L}_{\rho} \bar{w} m_{\rho}=\int_{\mathbb{R}^{3}}(\phi-\chi \bar{w}) m_{\rho},
$$

hence

$$
\int_{\mathbb{R}^{3}} \phi m_{\rho}=\int_{\text {supp } \chi} \chi \bar{w} m_{\rho} \leq C,
$$

where $C$ is a positive constant independent of $\rho$. From (2.18), since

$$
1=\int_{B_{R_{N}}} m_{\rho}+\int_{B_{R_{N}}^{C}} m_{\rho}
$$

then

$$
\int_{B_{R_{N}}} m_{\rho} \geq 1-\frac{C}{N}
$$


and from the convergence of $m_{\rho}$

$$
\int_{B_{R_{N}}} m \geq 1-\frac{C}{N}
$$

hence letting $N \rightarrow+\infty$ we obtain $\int_{\mathbb{R}^{3}} m=1$.

To prove that $\mathcal{L}^{*} m=0$ we have, for any $\psi$ smooth,

$$
0=\int_{\mathbb{R}^{3}}\left(\mathcal{L}_{\rho}^{*} m_{\rho}\right) \psi=\int_{\mathbb{R}^{3}}\left(\mathcal{L}_{\rho} \psi\right) m_{\rho} \rightarrow \int_{\mathbb{R}^{3}} \mathcal{L} \psi m=\int_{\mathbb{R}^{3}} \mathcal{L}^{*} m \psi,
$$

where we take account that $\mathcal{L}_{\rho} \psi \rightarrow \mathcal{L} \psi$ strongly and that $m_{\rho} \rightarrow m$.

We observe that, for $b \in C^{\infty}$, the uniqueness of the invariant measure comes from the results of Arnold, Klieman [5], or Ichihara, Kunita [22] by probabilistic methods. Under our assumptions, and using only analytical tools, the uniqueness of the invariant measure is established in Corollary 4.1 below.

Proposition 2.2. Assume the hypotheses of Theorem 2.1 and also $b \in C^{\infty}$. Then the density $m$ of the invariant measure is a $C^{\infty}$ function. Moreover $m>0$ in $\mathbb{R}^{3}$.

Proof. We observe that $m$ satisfies

$$
\begin{aligned}
0=\mathcal{L}^{*} m & =-\sum_{i, j} \partial_{i j}\left(\left(\sigma \sigma^{T}\right)_{i j} m\right)+\sum_{i} \partial_{i}\left(b_{i} m\right) \\
& =-\operatorname{tr}\left(\sigma \sigma^{T} D^{2} m\right)+\sum_{i} \partial_{i}\left(b_{i} m\right) \\
& =-\operatorname{tr}\left(\sigma \sigma^{T} D^{2} m\right)+\sum_{i} b_{i} \partial_{i} m+\left(\sum_{i} \partial_{i} b_{i}\right) m .
\end{aligned}
$$

From the regularity of the solutions of the hypoelliptic operators (see [21]) we get $m \in C^{\infty}$.

Let us now prove that $m>0$, to this end we shall follow the arguments in [22, Prop. 6.1]. We assume by contradiction that $\exists x^{0}$ such that $m\left(x^{0}\right)=0$ then $-m$ attains the maximum value 0 . Then we can invoke Bony's Maximum Principle; indeed even though in [15, Corollary 3.1] the coefficient of the zeroth order term must be nonnegative, in our case this condition is not necessary because the maximum value is zero. Therefore we get $-m=0$ which gives the desired contradiction.

\section{A Liouville type result}

In this section, we establish a Liouville type result, which holds true not only in the Heisenberg setting but also for $\sigma$ whose columns satisfy the general Hörmander condition. This result will be stated in Proposition 3.1. Although in the proof of Theorems 4.1 and 4.2 below it will be applied to the particular case of a regular solution, Proposition 3.1 contains a general statement which has its own independent interest.

Let us first recall from [21] the definition of Hörmander condition. 
Definition 3.1. The vector fields $X_{j} \in C^{\infty}\left(\mathbb{R}^{N}\right), j=1, \ldots m$, satisfy the Hörmander condition if $X_{1}, \ldots X_{m}$ and their commutators of any order span $\mathbb{R}^{N}$ at each point of $\mathbb{R}^{N}$.

Proposition 3.1. Consider the problem

$$
\mathcal{L} V=-\operatorname{tr}\left(\sigma(x) \sigma^{T}(x) D^{2} V\right)-b(x) \cdot D V=0, \quad x \in \mathbb{R}^{N}
$$

where $b$ is a globally Lipschitz continuous function in $\mathbb{R}^{N}$ and the vector fields $X_{j}=\sigma^{j} \cdot \nabla, j=1, \ldots m$ satisfy the Hörmander condition as in Definition 3.1. Assume that there exist $w \in C^{\infty}\left(\mathbb{R}^{N}\right)$ and $R_{0}>0$ such that

$$
\mathcal{L} w \geq 0 \quad \text { in } \overline{B\left(0, R_{0}\right)}{ }^{C}, \quad w(x) \rightarrow+\infty \quad \text { as }|x| \rightarrow+\infty .
$$

Then:

(i) every viscosity subsolution $V \in U S C\left(\mathbb{R}^{N}\right)$ to (3.1) such that $\lim \sup _{|x| \rightarrow+\infty} \frac{V}{w} \leq 0$ is constant;

(ii) every viscosity supersolution $V \in L S C\left(\mathbb{R}^{N}\right)$ to (3.1) such that $\liminf |x| \rightarrow+\infty \frac{V}{w} \geq 0$ is constant.

Proof. The proof uses the same arguments as in [28] (see also [7, Lemma 4.1 and remark 4.1]). For the sake of completeness, we shall give the proof of case (i); being similar, the proof of case (ii) is omitted.

Let us first observe that if $\psi \in \mathcal{C}^{2}(A)\left(A\right.$ is any open set $A \subset \overline{B\left(0, R_{0}\right)}{ }^{C}$ ) is a classical supersolution in A, i.e.

$$
\mathcal{L} \psi \geq 0 \text { in } A
$$

then $w+\psi$ is a viscosity supersolution in A, i.e.

$$
\mathcal{L}(w+\psi) \geq 0 \text { in } A \text {. }
$$

Define for each $\eta>0$ :

$$
V_{\eta}(x):=V(x)-\eta w(x) .
$$

We claim that $V_{\eta}$ is a viscosity subsolution in ${\overline{B\left(0, R_{0}\right)}}^{C}$ i.e.

$$
\mathcal{L}\left(V_{\eta}\right) \leq 0 \text { in } \overline{B\left(0, R_{0}\right)} \text {. }
$$

Indeed, let us assume by contradiction that there exists $\psi \in \mathcal{C}^{2}\left({\overline{B\left(0, R_{0}\right)}}^{C}\right)$ such that $V_{\eta}-\psi$ attains a strict maximum in some point $\bar{x} \in \overline{B\left(0, R_{0}\right)}$, $V(\bar{x})=\eta w(\bar{x})+\psi(\bar{x})$, and that there holds

$$
\mathcal{L}(\psi)(\bar{x})>0 .
$$

By the the continuity of the coefficients of $\mathcal{L}$, and the regularity of $\psi$ there exists a $r_{0}>0$ such that

$$
\mathcal{L}(\psi)(x)>0 \text { in } B\left(\bar{x}, r_{0}\right) \subset \overline{B\left(0, R_{0}\right)} \text {. }
$$

As remarked above $\eta w+\psi$ is a supersolution in $B\left(\bar{x}, r_{0}\right)$. Moreover there exists $\alpha>0$ such that $V(x)<\eta w(x)+\psi(x)-\alpha$ for any $x \in \partial B\left(\bar{x}, r_{0}\right)$. Then by a local comparison principle (see [8]), $V(x) \leq \eta w(x)+\psi(x)-\alpha$ in $B\left(\bar{x}, r_{0}\right)$ and for $x=\bar{x}$ we get a contradiction; hence our claim (3.3) is proved. 
Thanks to $V_{\eta} \rightarrow-\infty$ as $|x| \rightarrow+\infty$, there exists $R_{1}(\eta)=R_{1}>R_{0}$ such that

$$
V_{\eta}(x) \leq \sup _{|z|=R_{0}} V_{\eta}(z), \quad \forall|x| \geq R_{1}
$$

then, using the weak maximum principle applied to $V_{\eta}$,

$$
\max _{B\left(0, R_{1}\right) \backslash \overline{B\left(0, R_{0}\right)}} V_{\eta}=\max _{\partial B\left(0, R_{0}\right)} V_{\eta}
$$

and this implies that

$$
V_{\eta}(x) \leq \max _{\partial B\left(0, R_{0}\right)} V_{\eta}, \quad \forall x \in \overline{B\left(0, R_{0}\right)}{ }^{C} .
$$

Letting $\eta \rightarrow 0$ in the preceding inequality:

$$
V(x) \leq \max _{\partial B\left(0, R_{0}\right)} V, \quad \forall x \in{\overline{B\left(0, R_{0}\right)}}^{C} .
$$

Therefore $V$ attains its global maximum so it is a constant by the strong maximum principle established by Bardi and Da Lio [8, Corollary 3.2].

Remark 3.1. Note that, in the Heisenberg group, the function $w$ introduced in Lemma 2.2 satisfies assumptions (3.2).

Remark 3.2. Let us stress that the above arguments work for any linear operator $\mathcal{L}$ satisfying a strong maximum principle.

Remark 3.3. Note that conditions on the sub and super solutions in (i) and (ii) imply the boundedness of the sub and super solutions.

\section{Applications}

In this section we provide two applications of the previous results. In both cases we will use the existence of the invariant measure for the process (1.1) proved in Sect. 2 and the Liouville type property obtained in Sect. 3. Summarizing, we shall prove that

$$
\lim _{\delta \rightarrow 0^{+}} \delta u_{\delta}(x)=\lim _{t \rightarrow+\infty} u(t, x)=\lim _{t \rightarrow+\infty} \frac{v(t, x)}{t}=\int_{\mathbb{R}^{3}} f d m,
$$

where $m$ is the invariant measure of Sect. 2 and $u_{\delta}, u$ and $v$ are the solutions respectively of

$$
\begin{aligned}
& \delta u_{\delta}+\mathcal{L} u_{\delta}=f(\cdot), \quad \text { in } \mathbb{R}^{3}, \\
& u_{t}+\mathcal{L} u=0 \quad \text { in }(0,+\infty) \times \mathbb{R}^{3}, \quad u(0, \cdot)=f(\cdot) \quad \text { on } \mathbb{R}^{3}, \\
& v_{t}+\mathcal{L} v=f(\cdot) \quad \text { in }(0,+\infty) \times \mathbb{R}^{3}, \quad v(0, \cdot)=0 \quad \text { on } \mathbb{R}^{3},
\end{aligned}
$$

and $\mathcal{L}$ is the infinitesimal generator of the process (1.1), i.e. the operator defined in (1.4). 


\subsection{The ergodic problem}

In this section we tackle the following ergodic problem. We consider the family of problems

$$
\delta u_{\delta}(x)-\operatorname{tr}\left(\sigma(x) \sigma^{T}(x) D^{2} u_{\delta}\right)-b(x) D u_{\delta}=f(x) \quad \text { in } \mathbb{R}^{3},
$$

where $\delta>0$ and we investigate about the convergence as $\delta \rightarrow 0$ of $\delta u_{\delta}$ to a constant $\lambda$ called the ergodic constant. Throughout this section, we assume $\left(A_{1}\right) \sigma$ is defined in (1.2);

$\left(A_{2}\right) b \in C^{\infty}\left(\mathbb{R}^{3}\right)$ and satisfies the hypotheses of Lemma 2.2;

$\left(A_{3}\right) f \in C^{0}\left(\mathbb{R}^{3}\right) \cap L^{\infty}\left(\mathbb{R}^{3}\right)$.

The next two Lemma contain several properties of $u_{\delta}$ which will be used later on.

Lemma 4.1. Under Assumptions $\left(A_{1}\right)-\left(A_{3}\right)$, there exists an unique smooth viscosity solution $u_{\delta}$ of the approximating problem (4.1) such that

$$
\left|u_{\delta}(x)\right| \leq \frac{C}{\delta}, \quad \forall x \in \mathbb{R}^{3},
$$

for some positive constant $C$ independent of $\delta$.

Proof. The uniqueness follows from the comparison principle proved in [15]. By Assumption $\left(A_{3}\right)$ it is easy to see that $w^{ \pm}= \pm \frac{C}{\delta}$ with $C$ sufficiently large is respectively a supersolution and a subsolution for problem (4.1). In conclusion, applying Perron's method, we infer the existence of a solution to (4.1) verifying (4.2). Finally, the regularity of $u_{\delta}$ follows from hypoellipticity.

Lemma 4.2. Under Assumptions $\left(A_{1}\right)-\left(A_{3}\right)$, the functions $v_{\delta}:=\delta u_{\delta}$, where $u_{\delta}$ is the solution of problem (4.1), are locally uniformly Hölder continuous. Namely, there exists $\alpha \in(0,1)$ such that for every compact $K \subset \mathbb{R}^{3}$ there exists a constant $N$ such that

$$
\left|v_{\delta}\left(x_{1}\right)-v_{\delta}\left(x_{2}\right)\right| \leq N\left|x_{1}-x_{2}\right|^{\alpha}, \quad \forall x_{1}, x_{2} \in K, \forall \delta \in(0,1) .
$$

The constant $N$ only depends on $K$ and on the data of the problem (in particular is independent of $\delta$ ).

Proof. The statement is a direct consequence of the result of Krylov [24]. For the sake of completeness let us sketch how to apply Krylov's result to our case. From Lemma 4.1 the function $v_{\delta}$ is uniformly bounded and smooth and solves the following equation

$$
\delta v_{\delta}-\operatorname{tr}\left(\sigma(x) \sigma^{T}(x) D^{2} v_{\delta}\right)-b(x) D v_{\delta}=\delta f(x) \text { in } \mathbb{R}^{3} .
$$

We observe that Eq. (4.4) can be written in the form

$$
-L_{0} v_{\delta}+v_{\delta}:=-\sigma^{i k} \partial_{x_{i}}\left(\sigma^{j k} \partial_{x_{j}} v_{\delta}\right)-B D v_{\delta}+v_{\delta}=\delta f+(1-\delta) v_{\delta}
$$

where $B_{j}=b_{j}-\sum_{j k} \sigma^{i k} \partial_{x_{i}} \sigma^{j k}$ and $L_{0}=\sigma^{i k} \partial_{x_{i}}\left(\sigma^{j k} \partial_{x_{j}}{ }^{\cdot}\right)$.

For $\delta$ fixed, consider the problem

$$
\begin{cases}-L_{0} v_{\delta, n}+v_{\delta, n}=\delta f+(1-\delta) v_{\delta} & \text { in } B(0, n) \\ v_{\delta, n}=0 & \text { on } \partial B(0, n) .\end{cases}
$$


We observe that $\left\{v_{\delta, n}\right\}$ is a equibounded family (by the same arguments of Lemma 4.1). [24, Theorem 2.1] of Krylov ensures that there exists $\alpha \in(0,1)$ such that for every compact $K \subset \mathbb{R}^{3}$ there exists a constant $N_{1}$ (independent of $\delta, n)$ such that

$$
\left|v_{\delta, n}\left(x_{1}\right)-v_{\delta, n}\left(x_{2}\right)\right| \leq N_{1}\left|x_{1}-x_{2}\right|^{\alpha}, \quad \forall x_{1}, x_{2} \in K
$$

By Ascoli-Arzelà Theorem, letting $n \rightarrow+\infty$ (possibly passing to a subsequence) we get that $v_{\delta, n}$ converges locally uniformly to a function $V_{\delta}$. By the stability and uniqueness results we infer $V_{\delta}=v_{\delta}$. Moreover, passing to the limit in $n$ in (4.6), we get (4.3).

In the next result we prove that $\delta u_{\delta}$ converges to a constant which will be characterize in terms of the invariant measure of the process (1.1).

Theorem 4.1. Under Assumptions $\left(A_{1}\right)-\left(A_{3}\right)$, the solution $u_{\delta}$ of problem (4.1) given in Lemma 4.1 satisfies

$$
\lim _{\delta \rightarrow 0} \delta u_{\delta}=\int_{\mathbb{R}^{3}} f(x) d m(x), \text { locally uniformly }
$$

where $m$ is the invariant measure of process (1.1) founded in Sect. 2.

Proof. We shall proceed following some arguments of [7]. The functions $v_{\delta}:=$ $\delta u_{\delta}$ solve (4.4) and, from estimate (4.2), satisfy

$$
\left|v_{\delta}\right| \leq C, \quad \text { in } \mathbb{R}^{3}
$$

with $C$ independent of $\delta$, hence they are uniformly bounded in $\mathbb{R}^{3}$. From Lemma $4.2 v_{\delta}$ are also uniformly Hölder continuous in any compact set of $\mathbb{R}^{3}$. Then by the Ascoli-Arzelà theorem there is a sequence $\delta_{n} \rightarrow 0$ and a continuous function $w$ such that $v_{\delta_{n}} \rightarrow v$ locally uniformly; by stability, $v$ is a solution of

$$
-\operatorname{tr}\left(\sigma(x) \sigma^{T}(x) D^{2} v\right)-b(x) D v=0, x \in \mathbb{R}^{3},
$$

hence $v \in C^{\infty}$ by the hypoellipticity of the operator (see [15]). Then by Proposition 3.1, $v$ is constant.

In conclusion, we have that, possibly passing to a subsequence, $\left\{\delta u_{\delta}\right\}_{\delta}$ converges locally uniformly to a constant. Now, it remains to prove that this constant is independent of the subsequence chosen and that is has the form (4.7). By standard arguments of optimal control theory (see [19]), the function $u_{\delta}$ can be written as

$$
u_{\delta}(x)=\mathbb{E}_{x} \int_{0}^{+\infty} f\left(X_{t}\right) e^{-\delta t} d t
$$

where $X_{t}$ is the process in (1.1) with initial data $X_{0}=x$ while $\mathbb{E}$ denotes the expectation. Integrating both sides with respect to the invariant measure, we 
infer

$$
\begin{aligned}
\int_{\mathbb{R}^{3}} u_{\delta}(x) d m(x) & =\int_{0}^{+\infty}\left(\mathbb{E}_{x} \int_{\mathbb{R}^{3}} f\left(X_{t}\right) d m(x)\right) e^{-\delta t} d t \\
& =\int_{0}^{+\infty}\left(\int_{\mathbb{R}^{3}} f(x) d m(x)\right) e^{-\delta t} d t \\
& =\frac{1}{\delta} \int_{\mathbb{R}^{3}} f(x) d m(x)
\end{aligned}
$$

where the second equality is due to the definition of invariant measure. Taking into account that every convergent subsequence of $\left\{\delta u_{\delta}\right\}_{\delta}$ must converge to a constant, we conclude that the whole sequence $\left\{\delta u_{\delta}\right\}_{\delta}$ converges to $\int_{\mathbb{R}^{3}} f d m$.

Remark 4.1. As kindly suggested by the referee, we note that, with probabilistic methods and a suitable change of variables in (4.10), arguing as in [22, Proposition 5.1], we can obtain the result of Theorem 4.1. More precisely, from (4.10) we have

$$
\delta u_{\delta}(x)=\delta \int_{0}^{+\infty} \mathbb{E}_{x}\left[f\left(X_{t}\right)\right] e^{-\delta t} d t .
$$

Noting that $\mathbb{E}_{x}\left[f\left(X_{t}\right)\right]$ is the solution of $w_{t}+\mathcal{L} w=0, w(0, x)=f(x)$, then

$$
\delta u_{\delta}(x)=\delta \int_{0}^{+\infty} \int_{\mathbb{R}^{3}} P_{t}(x, y) f(y) e^{-\delta t} d y d t
$$

where $P_{t}(x, y)$ is the fundamental solution of the problem. Hence, by the change of variable $s=\delta t$, we have

$$
\delta u_{\delta}(x)=\int_{0}^{+\infty} e^{-s} \int_{\mathbb{R}^{3}} P_{s / \delta}(x, y) f(y) d y d s .
$$

Finally, using [22, Proposition 5.1 and Proposition 6.1] we obtain the convergence as in (4.7).

\subsection{Large time behavior of solutions}

This section concerns the asymptotic behavior for large time of the solution of the parabolic Cauchy problem:

$$
\begin{cases}u_{t}+\mathcal{L} u=0 & \text { in }(0,+\infty) \times \mathbb{R}^{3} \\ u(0, x)=f(x) & \text { on } \mathbb{R}^{3}\end{cases}
$$

where $\mathcal{L}$ is the operator defined in (1.4). Let us recall that, for periodic fully nonlinear equations, this issue was studied in [1, Theorem 4.2]. We quote here also the results in the manuscript [28].

Theorem 4.2. Under the assumptions of Theorem 2.1 and Proposition 3.1, for $f \in C^{0}\left(\mathbb{R}^{3}\right) \cap L^{\infty}\left(\mathbb{R}^{3}\right)$, the solution $u$ of problem (4.11) verifies

$$
\lim _{t \rightarrow+\infty} u(t, x)=\int_{\mathbb{R}^{3}} f(x) d m(x), \quad \text { locally uniformly in } x,
$$

where $m$ is the invariant measure of process (1.1) given in Sect. 2. 
Proof. Since $\pm\|f\|_{\infty}$ are sub and supersolution of (4.11), by the comparison principle we have that

$$
\|u\|_{\infty} \leq\|f\|_{\infty}
$$

Arguing as in [1, Theorem 4.2] we get, for some $c>0,|u(t+s, x)-u(t, x)| \leq c s$, and in particular $\left|u_{t}(t, x)\right| \leq c$. Moreover classical results on regularity of subelliptic operators give that $u(t, \cdot)$ are locally Hölder continuous on $x$ uniformly in $t$ (see $[15,24])$. Hence by Ascoli-Arzelà theorem for any sequence $t_{n} \rightarrow+\infty$ there exists a subsequence $t_{n_{k}}$ such that $u\left(t_{n_{k}}, \cdot\right) \rightarrow v$ locally uniformly for some $v \in C^{0}\left(\mathbb{R}^{3}\right)$. By standard arguments (see [1, Theorem 4.2]), $v$ is the solution of $\mathcal{L} v=0$; hence, by Proposition 3.1 (the Liouville type result), it is a constant. Therefore, we have

$$
u\left(t_{n_{k}}, \cdot\right) \rightarrow C \text {, locally uniformly . }
$$

We show now that the constant $C$ is independent of the chosen sequence. Let us consider an arbitrary sequence $\left\{s_{n}\right\}$ such that $s_{n} \rightarrow+\infty$ and $u\left(s_{n}, \cdot\right) \rightarrow C$ locally uniformly. From (1.3)

$$
\int_{\mathbb{R}^{3}} u\left(s_{n}, x\right) d m(x)=\int_{\mathbb{R}^{3}} f(x) d m(x)
$$

Using (4.12), $\int_{\mathbb{R}^{3}} d m(x)=1$ and the dominated convergence theorem, we get

$$
C=\int_{\mathbb{R}^{3}} f(x) d m(x) .
$$

Corollary 4.1. Under assumptions of Theorem 2.1 there exists at most one invariant probability measure $m$ for the process (1.1).

Proof. If, by contradiction, there are two measures $m_{1}$ and $m_{2}$, then from Theorem 4.2

$$
\int_{\mathbb{R}^{3}} f(x) d m_{1}(x)=\int_{\mathbb{R}^{3}} f(x) d m_{2}(x)
$$

for any $f(x) \in C^{0}\left(\mathbb{R}^{3}\right) \cap L^{\infty}\left(\mathbb{R}^{3}\right)$; hence, $m_{1}=m_{2}$.

Remark 4.2. We observe that for $b \in C^{\infty}$ the result of Theorem 4.2 it has already been obtained in [22, Proposition 5.1] with probabilistic methods.

Remark 4.3. Let us consider the following Cauchy problems

$$
\left\{\begin{array}{l}
v_{t}+\mathcal{L} v=f \text { in }(0,+\infty) \times \mathbb{R}^{3} \\
v(0, x)=0 \text { on } \mathbb{R}^{3},
\end{array}\right.
$$

where $\mathcal{L}$ is the operator defined in (1.4) and $f$ is a function as in Theorem 4.2.

By means of the Duhamel formula and a change of variables the solution $v$ can be written as $v(t, x)=\int_{0}^{t} u(\tau, x) d \tau$ where $u$ is the solution of (4.11). Hence the statement of Theorem 4.2 can be rephrased as

$$
\lim _{t \rightarrow+\infty} \frac{v(t, x)}{t}=\int_{\mathbb{R}^{3}} f(x) d m(x) .
$$




\section{The general case}

In this paragraph we highlight the general method (see [27]) which we applied so far within the framework of Heisenberg case. We address the stochastic dynamics (1.1) under the following assumptions:

$$
\begin{aligned}
& \left\{\begin{array}{l}
\sigma(x) \text { a real } N \times m \text { matrix with } \sigma \in C^{\infty}\left(\mathbb{R}^{N}\right), \\
\|\sigma(x)\| \leq C(|x|+1), \text { for some } C>0, \\
\text { the columns of } \sigma \text { satisfy Hörmander condition (see Definition 3.1); }
\end{array}\right. \\
& b \in C^{\infty}\left(\mathbb{R}^{N}\right),\|b(x)\| \leq C(|x|+1), C>0 ; \\
& \left\{\begin{array}{l}
\text { for } A:=\sigma \sigma^{T}, \text { there exists }\left\{A_{\rho}(x)\right\}_{\rho \in(0,1)} \text { with } A_{\rho}=\sigma_{\rho} \sigma_{\rho}^{T}, \\
\sigma_{\rho} \in C^{\infty}\left(\mathbb{R}^{N}\right), A_{\rho} \rightarrow A \text { in } L^{\infty} \text { and } A_{\rho} \text { is locally definite positive. }
\end{array}\right.
\end{aligned}
$$

There exists a function $w$ which verifies (2.9) for any $\rho$ sufficiently small.

The growth assumptions on $\sigma$ in (5.1) and on $b$ in (5.2) allow us to obtain the existence of a process $X_{t}$ in (1.1).

Under assumptions (5.1), (5.2), the Liouville type result contained in Proposition 3.1 still holds true. In fact the results of Bony [15] on comparison principle and strong maximum principle hold also in this setting if we observe that

$$
-\operatorname{tr}\left(\sigma \sigma^{T} D^{2} u\right)=\sum_{j} X_{j}^{2} u-C(x) \cdot D u,
$$

where $C(x)=D \sigma^{j} \cdot \sigma^{j}$ and $\sigma^{j}$ are the columns of the matrix $\sigma$.

Theorem 5.1. Under assumptions (5.1)-(5.4) there exists an invariant probability measure $m$ associated to the diffusion process (1.1).

Proof. We observe that, by assumptions (5.3), (5.4), there exists an unique invariant measure $m_{\rho}$ for the process with diffusion $\sigma_{\rho}$. Then, arguing as in the proof of Theorem 2.1, using again the function $w$ in (5.4) we obtain the existence of the invariant measure associated to the process (1.1).

Corollary 5.1. Under assumptions (5.1)-(5.4) and $f \in C^{0}\left(\mathbb{R}^{N}\right) \cap L^{\infty}\left(\mathbb{R}^{N}\right)$, Theorem 4.2, Corollary 4.1 and Theorem 4.1 hold true.

Remark 5.1. We observe that, as for the Heisenberg case, in many settings it suffices to require only $b$ Lipschitz continuous (see for instance Example 5.1 below).

A simple application of the general result is the case of Grushin operator.

Example 5.1. The Grushin operator

For $x=\left(x_{1}, x_{2}\right) \in \mathbb{R}^{2}$, consider the diffusion matrix

$$
\sigma(x)=\left(\begin{array}{cc}
1 & 0 \\
0 & x_{1}
\end{array}\right)
$$

and observe that $\sigma$ satisfies (5.1); actually, $X_{1}=(1,0), X_{2}=\left(0, x_{1}\right)$ and $\left[X_{1}, X_{2}\right]=(0,1)$ span all $\mathbb{R}^{2}$. In this case the infinitesimal generator is

$$
\mathcal{L} V=-V_{x_{1} x_{1}}-x_{1}^{2} V_{x_{2} x_{2}}-b(x) \cdot D V .
$$


Moreover

$$
\sigma_{\rho}(x)=\left(\begin{array}{ccc}
1 & 0 & 0 \\
0 & x_{1} & \rho
\end{array}\right)
$$

satisfies (5.3).

We take $b(x)=\left(b_{1}\left(x_{1}\right), b_{2}\left(x_{2}\right)\right)$ satisfying (2.7) and (5.2) and $w(x)=$ $\frac{1}{12} x_{1}^{4}+\frac{1}{2} x_{2}^{2}$. Then $\mathcal{L} w \geq 1$ is equivalent to the following condition

$$
-\frac{1}{3} b_{1}\left(x_{1}\right) x_{1}^{3}-b_{2}\left(x_{2}\right) x_{2} \geq 2 x_{1}^{2}+1 .
$$

We want to prove (5.6) for $\max \left\{\left|x_{1}\right|,\left|x_{2}\right|\right\} \geq \tilde{R}$, with $\tilde{R}$ sufficiently large.

Arguing as in the proof of Lemma 2.2 we consider two cases: (1) $\left|x_{1}\right|>\tilde{R},(2)$ $\left|x_{1}\right| \leq \tilde{R}$ and $\left|x_{2}\right|>\tilde{R}$.

(1) If $\left|x_{1}\right|>\tilde{R}$, we have two possibilities: (1a) $\left|x_{2}\right|>R$, or (1b) $\left|x_{2}\right| \leq R$.

(1a) We consider $x_{2}>R$ (the case $x_{2}<-R$ is analogous). From (2.7),

$$
-\frac{1}{3} b_{1}\left(x_{1}\right) x_{1}^{3}-b_{2}\left(x_{2}\right) x_{2} \geq C_{1} \frac{x_{1}^{2+\alpha}}{3}+C_{2} x_{2}^{\alpha} \geq C_{1} \frac{x_{1}^{2+\alpha}}{3} \text {. }
$$

Then we get (5.6) provided $C_{1} \geq \frac{6}{\tilde{R}^{\alpha}}+\frac{3}{\tilde{R}^{2+\alpha}}$.

(1b) If $\left|x_{1}\right|>\tilde{R}$ and $\left|x_{2}\right| \leq R$, setting $M_{2}:=\max _{\left|x_{2}\right| \leq R}\left|b_{2}\left(x_{2}\right) x_{2}\right|$,

$$
-\frac{1}{3} b_{1}\left(x_{1}\right) x_{1}^{3}-b_{2}\left(x_{2}\right) x_{2} \geq C_{1} \frac{x_{1}^{2+\alpha}}{3}-M_{2} .
$$

Then we get (5.6) provided $C_{1} \geq \frac{6}{\tilde{R}^{\alpha}}+\frac{1+M_{2}}{\tilde{R}^{2+\alpha}}$.

(2) If $\left|x_{1}\right| \leq \tilde{R}$ and $x_{2}>\tilde{R}$ (the case $x_{2}<-R$ is analogous), we consider the two subcases: $2 \mathrm{a})\left|x_{1}\right| \leq R$ or (2b) $R<x_{1}<\tilde{R}$.

(2a) If $\left|x_{1}\right| \leq R$, we set $M_{1}:=\max _{\left|x_{1}\right| \leq R}\left[\frac{1}{3} b_{1}\left(x_{1}\right) x_{1}^{3}+2 x_{1}^{2}+1\right]$. Since $-b_{2}\left(x_{2}\right)$ $x_{2} \geq C_{2} x_{2}^{\alpha}$, then (5.6) holds true provided $C_{2} \geq \frac{M_{1}}{\tilde{R}^{\alpha}}$.

(2b) If $x_{2}>\tilde{R}$ with $R>1, R<x_{1}<\tilde{R}$, we have

$$
-\frac{1}{3} b_{1}\left(x_{1}\right) x_{1}^{3}-b_{2}\left(x_{2}\right) x_{2} \geq C_{1} \frac{x_{1}^{2+\alpha}}{3}+C_{2} x_{2}^{\alpha} .
$$

Hence, (5.6) holds provided $C_{1} \geq 9$ since $-C_{1} \frac{x_{1}^{2+\alpha}}{3}+2 x_{1}^{2}+1$ becomes negative.

Remark 5.2. Lions-Musiela in [28] considered a similar degenerate case but in their paper the elements of the matrix $\sigma(x)$ are bounded in $\mathbb{R}^{2}$, i.e. they considered

$$
\sigma(x)=\left(\begin{array}{cc}
1 & 0 \\
0 & \frac{x_{1}}{\sqrt{1+x_{1}^{2}}}
\end{array}\right)
$$

\section{A Appendix}

In the following lemma we state the equivalence between conditions (2.9) and (2.10) when the coefficients of the operator are smooth. This property has already been established by Lions [27]; however, for the sake of completeness we shall provide the proof. 
Lemma A.1. Consider a linear operator

$$
\mathcal{G}(u):=-\operatorname{tr}\left(\tau \tau^{T} D^{2} u\right)-\beta \cdot D u
$$

where $\tau$ is a matrix whose columns verify the Hörmander condition (see Definition 3.1), $\tau$ and $\beta$ are smooth functions with

$$
|\tau(x)|,|\beta(x)| \leq C(1+|x|) \quad \forall x \in \mathbb{R}^{N} .
$$

Then, conditions (2.9) and (2.10) are equivalent; namely the following properties are equivalent:

(i) there exists $w \in C^{\infty}\left(\mathbb{R}^{N}\right)$ such that

$$
\mathcal{G}(w) \geq 1 \quad \text { in } \overline{B\left(0, R_{0}\right)}{ }^{C}, \quad w \geq 0 \quad \text { in } \overline{B\left(0, R_{0}\right)}{ }^{C}, \quad \lim _{|x| \rightarrow+\infty} w=+\infty
$$

for some constant $R_{0}>0$;

(ii) there exists $\bar{w} \in C^{\infty}\left(\mathbb{R}^{N}\right)$ such that

$$
\mathcal{G}(\bar{w})+\chi \bar{w}=\phi \quad \text { in } \mathbb{R}^{N}, \quad \lim _{|x| \rightarrow+\infty} \bar{w}=+\infty
$$

for some $C^{\infty}$ functions $\chi$ and $\phi$ with $\lim _{|x| \rightarrow+\infty} \phi=+\infty, \chi \geq 0$ and supp $\chi$ compact.

Proof. For completeness, we report the arguments of [27]. As one can easily check, property (ii) obviously implies property (i) (possibly adding a constant).

Now, assuming (i), we want to prove (ii). We denote $K:=\frac{\max }{B\left(0, R_{0}\right)}|w|$ and $K_{\mathcal{G}}:=\frac{\max }{B\left(0, R_{0}\right)}|\mathcal{G}(w)|$. We fix $\chi \in C_{0}^{\infty}\left(\mathbb{R}^{N}\right)$ such that $\chi \geq 0, \chi=1$ in $B\left(0, R_{0}\right)$ and supp $\chi \subset B\left(0,2 R_{0}\right)$. We claim that the function $w^{b}(x):=w(x)+K+K_{\mathcal{G}}+1$ satisfies

$$
\mathcal{G}\left(w^{b}\right)+\chi w^{b}=: f^{*}(x) \geq 1 \quad \text { in } \mathbb{R}^{N}, \quad \lim _{|x| \rightarrow+\infty} w^{b}=+\infty .
$$

Indeed, the latter property is an immediate consequence of (i).

Moreover, for $|x| \leq R_{0}$, we have

$$
\mathcal{G}\left(w^{b}\right)+\chi w^{b} \geq-K_{\mathcal{G}}+\chi\left(w+K+K_{\mathcal{G}}+1\right) \geq 1
$$

while, for $|x| \geq R_{0}$, we have

$$
\mathcal{G}\left(w^{b}\right)+\chi w^{b} \geq \mathcal{G}(w) \geq 1
$$

hence, our claim (A.1) is proved.

Let us now consider a regular partition of unity $\left\{\phi_{i}\right\}_{i \geq 1}$ such that $\phi_{i} \geq 0$, $\sum_{i=1}^{\infty} \phi_{i}(x)=1, \operatorname{supp} \phi_{i} \subset \overline{B(0, i+1)} \backslash B(0, i-1)$. 
We claim that there exists a regular solution to

$$
\mathcal{G}\left(W_{n}\right)+\chi W_{n}=\sum_{i=1}^{n} \phi_{i} \quad \text { in } \mathbb{R}^{N}, \quad 0 \leq W_{n} \leq w^{b}
$$

In order to prove this existence, it is expedient to introduce, for $m \geq n+1$ and $\epsilon>0$, the following boundary value problems

$$
\begin{cases}(\mathcal{G}-\epsilon \Delta)\left(W_{n m}^{\epsilon}\right)+\chi W_{n m}^{\epsilon}=\sum_{i=1}^{n} \phi_{i} & \text { in } B(0, m) \\ W_{n m}^{\epsilon}=0 & \text { on } \partial B(0, m) .\end{cases}
$$

By the non-degeneracy of the operator, the comparison principle applies to problems (A.3). Hence, the Perron's method ensures that there exists a unique solution to (A.3). By standard arguments in hypoelliptic theory (see [24,32]), as $\epsilon \rightarrow 0^{+}, W_{n m}^{\epsilon}(x)$ converges to $W_{n m}(x)$ in $B(0, m)$, where $W_{n m}$ is the solution to

$$
\begin{cases}\mathcal{G}\left(W_{n m}\right)+\chi W_{n m}=\sum_{i=1}^{n} \phi_{i} & \text { in } B(0, m) \\ W_{n m}=0 & \text { on } \partial B(0, m),\end{cases}
$$

where the boundary condition is attained only in the viscosity sense. We observe that the Hörmander condition guarantees the comparison principle for (A.4); since 0 and $w^{b}$ are respectively a sub- and a supersolution, there holds true $0 \leq W_{n m} \leq w^{b}$ in $B(0, m)$. On the other hand, for $m_{1}>m$, still by comparison principle, we infer $W_{n m_{1}}^{\epsilon}(x) \geq W_{n m}^{\epsilon}(x)$ for every $x \in B(0, m)$; so, as $\epsilon \rightarrow 0^{+}$, we get $W_{n m_{1}}(x) \geq W_{n m}(x)$ for every $x \in B(0, m)$, namely, the sequence $\left\{W_{n m}\right\}_{m}$ is nondecreasing and locally bounded. Passing to the limit and using the regularity theory for hypoelliptic operators (see [15]), we accomplish the proof of our claim (A.2).

By (A.2), the functions $w_{i}(x):=W_{i}(x)-W_{i-1}(x)$ solve

$$
\mathcal{G}\left(w_{i}\right)+\chi w_{i}=\phi_{i} \quad \text { in } \mathbb{R}^{N}
$$

and verify: $\sum_{i=1}^{\infty} w_{i}(x)<\infty$ in $\mathbb{R}^{N}$.

Let us recall an elementary result: for any $\sum_{i=1}^{\infty} a_{i}<+\infty$ with $a_{i} \geq 0$, there exists a sequence $\left\{\lambda_{i}\right\}_{i}$ such that $\lim _{i \rightarrow+\infty} \lambda_{i}=+\infty$ and $\sum_{i=1}^{\infty} \lambda_{i} a_{i}<$ $+\infty$.

Then in our case there exists a sequence $\left\{\lambda_{i}\right\}_{i}$ such that $\lim _{i \rightarrow+\infty} \lambda_{i}=$ $+\infty$ and $\sum_{i=1}^{\infty} \lambda_{i} w_{i}(0)=K<+\infty$.

Let $n_{0} \in \mathbf{N}$ be fixed. Let us denote by $w_{n}^{\sharp}(x):=\sum_{i=1}^{n} \lambda_{i} w_{i}(x)$. In $B\left(0, n_{0}\right)$ $w_{n}^{\sharp}(x)$ satisfies: 


$$
\mathcal{G}\left(w_{n}^{\sharp}\right)+\chi w_{n}^{\sharp}=\sum_{i=1}^{n_{0}+1} \lambda_{i} \phi_{i} \quad w_{n}^{\sharp} \geq 0
$$

and by Harnack inequality (see [15]) there exists a constant $C_{n_{0}}$ independent of $n$ such that

$$
\begin{aligned}
\sup _{B\left(0, \frac{n_{0}}{2}\right)} w_{n}^{\sharp} & \leq C_{n_{0}}\left(\inf _{B\left(0, \frac{n_{0}}{2}\right)} w_{n}^{\sharp}+\sup _{B\left(0, \frac{n_{0}}{2}\right)} \sum_{i=1}^{n_{0}+1} \lambda_{i} \phi_{i}\right) \\
& \leq C_{n_{0}}\left(K+\sup _{B\left(0, \frac{n_{0}}{2}\right)} \sum_{i=1}^{n_{0}+1} \lambda_{i} \phi_{i}\right)=C_{n_{0}}^{*}
\end{aligned}
$$

This implies that in any bounded set $w^{\sharp}$ is well defined, i.e. $w^{\sharp}(x):=\sum_{i=1}^{\infty}$ $\lambda_{i} w_{i}(x)<\infty$ for every $x \in \mathbb{R}^{N}$. Moreover the function $w^{\sharp}$ satisfies

$$
\mathcal{G}\left(w^{\sharp}\right)+\chi w^{\sharp}=\sum_{i=1}^{\infty} \lambda_{i} \phi_{i}=: \phi, \quad w^{\sharp} \geq 0
$$

with $\lim _{x \rightarrow \infty} \phi(x)=+\infty$.

In conclusion, by (A.1) and (A.6), the function $\bar{w}:=w^{b}+w^{\sharp}$ satisfies (ii).

Remark A.1. In this proof we used the vanishing viscosity method because it also applies to more degenerate cases. As a matter of facts, one can easily obtain the existence of a continuous viscosity solution to (A.4) using the Perron's method and the strong Maximum Principle (see [8]).

Finally, let us stress that the smoothness of $\beta$ is needed only for applying the Harnack inequality.

\section{Acknowledgements}

We thank Italo Capuzzo Dolcetta and Olivier Ley for helpful discussions. We are grateful to the reviewers for the useful remarks and suggestions. The first and the second authors are members of GNAMPA-INdAM. The second author has been partially supported also by the "Progetto di Ateneo: Traffic flow on networks: analysis and control" of the University of Padova. The third author has been partially funded by the ANR project ANR-12-BS01-0008-01.

\section{References}

[1] Alvarez, O., Bardi, M.: Ergodicity, stabilization, and singular perturbations for Bellman-Isaacs equations. Mem. Am. Math. Soc. 204, 1-77 (2010)

[2] Alvarez, O., Bardi, M., Marchi, C.: Multiscale problems and homogenization for second-order Hamilton-Jacobi equations. J. Differ. Equ. 243, 349-387 (2007)

[3] Arapostathis, A., Borkar, V.S., Ghosh, M.K.: Ergodic control of diffusion processes. In: Encyclopedia of Mathematics and its Applications, vol. 143. Cambridge University Press (2012) 
[4] Arisawa, M., Lions, P.L.: On ergodic stochastic control. Commun. Partial Differ. Equ. 23, 2187-2217 (1998)

[5] Arnold, L., Kliemann, W.: On unique ergodicity for degenerate diffusions. Stochastics 21, 41-61 (1987)

[6] Bardi, M., Cesaroni, A.: Liouville properties and critical value of fully nonlinear elliptic operators. J. Differ. Equ. (2016). doi:10.1016/j.jde.2016.06.006

[7] Bardi, M., Cesaroni, A., Manca, L.: Convergence by viscosity methods in multiscale financial models with stochastic volatility. SIAM J. Financ. Math. 1, 230$265(2010)$

[8] Bardi, M., Da Lio, F.: Propagation of maxima and strong maximum principle for viscosity solutions of degenerate elliptic equations. I, Convex operators. Nonlinear Anal. 44, 991-1006 (2001)

[9] Basak, G.K., Borkar, V.S., Ghosh, M.K.: Ergodic control of degenerate diffusions. Stoch. Anal. Appl. 15, 1-17 (1997)

[10] Bensoussan, A., Lions, J.L.: Asymptotic Analysis for Periodic Structures. NorthHolland, Amsterdam (1978)

[11] Bensoussan, A.: Perturbation Methods in Optimal Control, Wiley/GauthierVillars Series in Modern Applied Mathematics. Wiley, Chichester, GauthierVillars, Montrouge (1988)

[12] Birindelli, I., Capuzzo Dolcetta, I., Cutrì, A.: Liouville theorems for semilinear equations on the Heisenberg group. Ann. Inst. H. Poincaré Anal. Non Linéaire 14, 295-308 (1997)

[13] Birindelli, I., Capuzzo Dolcetta, I., Cutrì, A.: Indefinite semi-linear equations on the Heisenberg group: a priori bounds and existence. Commun. Partial Differ. Equ. 23, 1123-1157 (1998)

[14] Biroli, M., Mosco, U., Tchou, N.: Homogenization by the Heisenberg group. Adv. Math. Sci. Appl. 7, 809-831 (1997)

[15] Bony, J.M.: Principe du maximum, inégalite de Harnack et unicité du probléme de Cauchy pour les opérateurs elliptiques dégénérés. Ann. Inst. Fourier (Grenoble) 19, 277-304 (1969)

[16] Capuzzo Dolcetta, I., Cutrì, A.: On the Liouville property for subLaplacians. Ann. Scuola Norm. Sup. Pisa Cl. Sci. 25(4), 239-256 (1997)

[17] Cirant, M.: On the solvability of some ergodic control problems. SIAM J. Control Optim. 52, 4001-4026 (2014)

[18] Dragoni, F., Kontis, V., Zegarlinski, B.: Ergodicity of Markov semigroups with Hörmander type generators in infinite dimensions. Potential Anal. 37, 199$227(2012)$

[19] Fleming, W.H., Soner, H.M.: Controlled Markov Processes and Viscosity Solutions. Springer, Berlin (1993) 
[20] Has'minskiü, R.Z.: Stochastic stability of differential equations. In: Monographs and Textbooks on Mechanics of Solids and Fluids: Mechanics and Analysis, vol. 7. Sijthoff \& Noordhoff, Alphen aan den Rijn-Germantown, Md. (1980)

[21] Hörmander, L.: Hypoelliptic second order differential equations. Acta Math. Upps. 119, 147-171 (1967)

[22] Ichihara, K., Kunita, H.: A classification of the second order degenerate elliptic operators and its probabilistic characterization. Z. Wahrscheinlichkeitstheorie und Verw. Gebiete 30, 235-254 (1974)

[23] Kabanov, Y.; Pergamenshchikov, S.: Two-scale stochastic systems. Asymptotic analysis and control. In: Applications of Mathematics (New York), Stochastic Modelling and Applied Probability, vol. 49. Springer, Berlin (2003)

[24] Krylov, N.V.: Hölder continuity and $L_{p}$ estimates for elliptic equations under general Hörmander's condition. Topol. Methods Nonlinear Anal. 9, 249258 (1997)

[25] Kunita, H.: Asymptotic behavior of the nonlinear filtering errors of Markov processes. J. Multivar. Anal. 1, 365-393 (1971)

[26] Kushner, H.J: Weak convergence methods and singularly perturbed stochastic control and filtering problems. Birkhäuser, Boston (1990)

[27] Lions, P.L.: Lectures at Collège de France 2014-2015. http://www. college-de-france.fr/site/pierre-louis-lions/course-2014-2015.htm. Accessed 10 July 2016

[28] Lions, P.L., Musiela, M.: Ergodicity of diffusion processes (unpublished)

[29] Lorenzi, L., Bertoldi, M.: Analytical Methods for Markov Semigroups. Chapman \& Hall/CRC, Boca Raton (2007)

[30] Mannucci, P., Marchi, C., Tchou, N.: Singular perturbations for some unbounded subelliptic operators (unpublished)

[31] Mannucci, P., Stroffolini, B.: Periodic homogenization under a hypoellipticity condition. NoDEA Nonlinear Differ. Equ. Appl. 22, 579-600 (2015)

[32] Oleinik, O.A., Radkevic, E.V.: Second Order Equations with Nonnegative Characteristic Form. Plenum Press, New York (1973)

Paola Mannucci

Dipartimento di Matematica

Università degli Studi di Padova

Via Trieste 63

35131 Padua

Italy

e-mail: mannucci@math.unipd.it 
Claudio Marchi

Dipartimento di Ingegneria dell'Informazione

Università degli Studi di Padova

Via Gradenigo 6

35131 Padua

Italy

e-mail: claudio.marchi@unipd.it

Nicoletta Tchou

IRMAR, Università de Rennes 1

Campus de Beaulieu

35042 Rennes Cedex

France

e-mail: nicoletta.tchou@univ-rennes1.fr

Received: 16 December 2015.

Accepted: 11 July 2016. 